\title{
Tangence
}

\section{La condition paradigmatique de la critique : le cas québécois}

\section{Nicole Fortin}

Numéro 51, mai 1996

Paradigmes critiques

URI : https://id.erudit.org/iderudit/025902ar

DOI : https://doi.org/10.7202/025902ar

Aller au sommaire du numéro

Éditeur(s)

Tangence

ISSN

0226-9554 (imprimé)

1710-0305 (numérique)

Découvrir la revue

Citer cet article

Fortin, N. (1996). La condition paradigmatique de la critique : le cas québécois. Tangence, (51), 8-27. https://doi.org/10.7202/025902ar d'utilisation que vous pouvez consulter en ligne.

https://apropos.erudit.org/fr/usagers/politique-dutilisation/ 


\section{La condition paradigmatique de la critique : le cas québécois}

Nicole Fortin

Sans doute n'est-ce pas seulement l'effet de quelque mode, ni de quelque élégance théorique, qui conduit la critique à penser de plus en plus les discours sous la forme unitaire du paradigme. Aujourd'hui, le mot se vêt du lustre du succès et, débordant l'explication restreinte du signe, vient englober tout l'espace des savoirs et des sens, déclinant en des termes nouveaux la cohérence des grands systèmes qu'une postmodernité régnante, suspicieuse et subversive a délestés de toutes aspirations historiques et structurales.

Certes, pareille postmodernité est loin d'être étrangère à l'engouement. L'on pourrait sans doute voir, dans ce paradigme mis au goût du jour, l'un de ses propres termes vecteurs, qui agit probablement moins comme énoncé théorique importé que comme forme figurative de ses enjeux: avant même de sémantiser la chose postmoderne, la vision paradigmatique du sens s'inscrit dans le mot en soi qui, par la contradiction entre post et moderme qu'il figurativise, se désigne formellement comme un lieu de distanciation, d'altérité, de recatégorisation, donc de posture et de travail paradigmatique ${ }^{1}$. Peut-être n'importe-t-il même pas que l'on juge — parfois simultanément d'ailleurs — la postmodernité comme manifestation ou comme éclatement de quelque paradigme possible: par delà les visions paradoxales qui l'entérinent ou le réprouvent tout à la fois, le concept de paradigme ne peut

1 D'autant plus que le jeu des déclinaisons paradigmatiques ne s'arrête pas là : le flou fréquemment maintenu entre le "postmoderne", la "postmodernité " ou le "postmodemisme" - avec ou sans le trait-d'union figurativisant la scission des deux temporalités inscrites dans le mot - ne s'apparente sans doute pas seulement à l'hésitation de parents querelleurs devant le prénom à donner à leur rejeton. Quiconque connaît l'esprit ludique des discoureurs de la postmodernité aura tôt fait de juger l'ambiguïté et l'instabilité voulues, voire amoureusement entretenues afin d'en faire des figures, des objets de discours sur l'identité même du paradigme: pareille imprécision maintient autour du signe une multiplicité de points de vue, d'écoles et d'origines (anglo-saxonne ou française) qui fractionnent et diffractent le sens. 
qu'opérer, compte tenu de ce que cette pratique se déploie en soi comme l'interrogation généralisée des systèmes, tour à tour reniés, éclatés, déviés, dédoublés ou parodiés. Que cette postmodernité soit une construction réelle ou fabulée ne change d'ailleurs rien à ses effets paradigmatiques et, dans un cas comme dans l'autre, le point de vue adopté pose comme valeur la recatégorisation du sens.

Dès lors, vouloir poser l'existence de cette postmodernité comme étant celle d'un paradigme inédit déboucherait vers une discussion qui tournerait rapidement à vide, engageant la recherche dans des parcours piégés, bâtis à l'image des labyrinthes jonchés de miroirs et de trompe-l'œil qu'une telle posture se complaît à dresser. Néanmoins, c'est là une des questions qui accaparera le plus le champ de discussion des postmodernes qui, d'une part, débattront de la possible cohérence d'une épistémè appelée postmodernité ou, d'autre part, chercheront sans cesse à situer cette dernière dans et par rapport aux autres paradigmes - soit au premier chef la modernité, cette altérité parfois jumelle par et contre laquelle elle se pose intrinsèquement. Ce flou formel ne saurait témoigner d'une impuissance du système à se fixer ou à atteindre un certain seuil de formalisation nécessaire. Ici, il serait plus juste de penser que la cohésion paradigmatique n'est pas tant un niveau de stabilité visé qu'un objet nécessaire de discours. De la sorte, la postmodernité régulariserait beaucoup mieux son champ en assurant la cohérence et le maintien des réflexions hétérogènes sur son identité épistémique qu'en fondant épistémologiquement cette dernière.

Par conséquent, il serait sans doute plus rentable de définir cette posture discursive actuelle non pas comme un paradigme-- qu'elle aurait tôt fait de disloquer et d'annihiler -, mais bien comme un lieu de mise en jeu de la condition paradigmatique. La postmodernité est, peut-on en poser l'hypothèse, un lieu (esthétique et théorique) d'exhibition et de mise à l'épreuve des grands procès de signification. Elle ne se déploie pas seulement comme mise en scène ou comme mise en accusation des grands et petits discours; plus largement, elle assigne à la barre les modes de signification, confrontant spectaculairement les systèmes paradigmatiques existants ou, encore, le signe avec son référent, l'objet avec son interprétant, le signe unique avec la série qui l'assume. "Le discours du post-moderne, disait Meschonnic, 
mime le post-moderne" ${ }^{2}$, dévoilant par là que le discours se construit, en ses niveaux figuratif, énonciatif, argumentatif, etc., sur la représentation même de son exercice. Là où la modernité s'était construite sur l'unité et l'autonomie du texte, la postmodernité se tourne plus globalement vers le procès du signe ou, plus précisément, vers les mécanismes de la construction du sens dont elle reproduit mimétiquement - et à outrance - les procédés et les résultats. Le versant le plus spéculaire de cette démarche réside sans doute dans la déconstruction, qui ressemble fort peu à une méthode et beaucoup à un vaste déploiement à l'infini de la logique sémiosique, où jamais un sens unique ne peut être arrêté, où jamais ne se marque la distance entre l'interprété et l'interprétation, où s'illustre en abyme le mouvement perpétuel et idéal de toute sémiosis ${ }^{3}$.

En quelque sorte, l'attitude postmoderne contribuerait à mimer la mécanique paradigmatique, tout en se désagrégeant, se minant et se niant elle-même comme paradigme stable. Â preuve, 1) le refus postmoderne de toute légitimation des codes normatifs et hégémoniques ou de tout esprit tribal, désintégrant dès lors tout "noyau dur" autour duquel pourrait se conformer l'existence et la cohérence des paradigmes - refus auquel s'ajoute la légitimation des petits récits marginaux, inclassables, de mauvais goût,

2 Henri Meschonnic, Modernité, modernité, Paris, Verdier, 1988, p. 246.

3 Dans Les limites de l'interprétation (Grasset, 1992), Umberto Eco pose d'ailleurs, en forme d'hypothèse, cet enjeu de figurativisation - mimétique ou parodique - du procès général de la signification (ici transcrit selon le modèle de Peirce): "Est-il légitime de dire que la dérive infinie dont parle la déconstruction est une forme de sémiosis illimitée au sens peircéen?" (p. 373). On pourrait aussi reprendre la définition donnée par Walter Moser qui, paraphrasant H.-U. Gumbrecht, "propose de comprendre la déconstruction comme une démythification seconde: quand la première démythification - sous la forme d'une critique moderne des préjugés et irrationalités - n'a pas mené à l'avènement d'une société heureuse en vertu de son état éclairé, l'activité critique s'est attaqué aux fondations conceptuelles mêmes (rationalité réflexive du sujet, transparence cognitive du langage, théorie de la représentation) de la première démythification. La déconstruction serait cette démythification au second degrế" ("Réinscrire, déconstruire: une critique du troisième type",

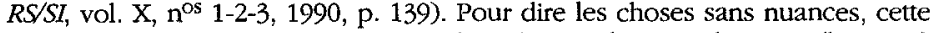
déconstruction pose moins, comme objet de son discours, le signe (littéraire) qu'un paradigme antécédent, dont elle entreprend de dénoncer et de réénoncer le fonctionnement. Selon cette citation, la déconstruction s'insère moins dans un rapport bistorique de succession que dans un rapport second (et critique) de signification par rapport au paradigme de la modernité, dont les mécanismes de fonctionnement deviennent l'objet de la mise en discours. 
démodés, "extra-paradigmatiques " ${ }^{4}$; 2) l'bybridation des formes et des sujets, concourant à démultiplier et à faire éclater la cohérence des énoncés et des énonciations en des signes et des voix multiples, pluri-identitaires, pluri-génériques et inassociables à quelque adresse paradigmatique centralisée et unique; 3 ) le refus de la scission entre le sujet et l'objet, soit des pôles construits de la signification, grâce à un court-circuitage de l'un et de l'autre qui abolit tout rapport interprété / interprétant usuel, donc toute illustration canonique de la dynamique du sens; 4) la confusion entre critique et création, qui ne concourt pas seulement à réduire la distance entre pratique et objet - ou entre lecture et écriture, métalangage et langage -, ni à indexer tout discours sous un régime fictionnel, mais aussi à affaiblir et à ironiser la dimension métadiscursive du discours, soit du lieu autonome de recatégorisation du sens; dans le même ordre d'idées, il faut noter le retour du lisible, qui annule toute hégémonie d'un lieu second de décodage; 5) le refus ou la fin de l'Histoire ou, plus justement, la destruction de toute cohésion ou évolution historique des paradigmes par le rabattement, sur le présent du discours, du lieu ultime de l'indexation et de la cohérence des discours du passé: au terme de cette marche arrière dans le courant successif de l'Histoire, on voit ces discours intégrés a posteriori, réécrits, redistribués et rebaptisés dans de grands unités intégratrices parfois ouvertement fictives, souvent étrangères à leur paradigme d'origine ${ }^{5}$;

4 Cette absence d'une bégémonie discursive devrait être relativisée à la lumière du "politically correct ", qui est à la fois ouverture sur les objets de discours - soit refus de toute exclusion par la valorisation et par l'égalisation de l'altérité, de la marginalisation, de l'hétérogène - et fermeture du sujet du discours - soit refus d'une autonomie du sujet comme de toutes paroles marginales, décentrées, hors d'une pensée homogénéisêe que l'on a idéalisée. Ici, le décalage "énoncé / énonciation" trouve aussi un de ces lieux de positionnement par le déphasage et le décalage posés entre un "réel "et une "transposition langagière du réel", centrés et construits à partir de structures hégémoniques distinctes.

5 Comme le formulera Lyotard en illustrant la recatégorisation inversée de cette logique épistémique, tout "ne peut devenir moderne que s'[il] est d'abord postmoderne" (Réponse à la question: qu'est-ce que le postmoderne?", Critique, $\mathrm{n}^{\circ} 419$, avril 1982, p. 365): semblable citation, énoncée autrement chez plus d'un, prend ici la forme du cliché stéréotypant, autour d'un syntagme unique, certaines des stratégies catégorielles de la postmodernité. Étant donné que la postmodernité se définit comme un lieu de "mise en scène" de ses propres mécanismes, il est significatif qu'elle synthétise autour d'énoncés-types les règles même de son fonctionnement. 
12

6) l'importance de la citation, de la récupération, du recyclage qui impliquent des paradigmes de reprise et de duplication, tout en abolissant cependant la portée historique de ces réénonciations dont on tait toute origine: l'ancienne et l'autre parole ne prennent pas seulement place aux côtés du nouveau; elles voient neutraliser toute ancienneté et toute altérité par leur rabattement, le plus souvent sous forme de discours indistinguables, dans un paradigme du présent et du pareil ; 7) dans la foulée, l'importance de l'intertextualité elle-même, qui rassemble les textes en un réseau figuré par des images telles la "bibliothèque" ou l'"encyclopédie", qui concourent à reconstruire métaphoriquement l'espace généralisé des discours; 8) l'importance de la carnavalisation, de la parodie, du simulacre qui ne présentent le signe que dans un lieu essentiellement décalé, transcodé, à double-sens, en retrait de toute structure dominante et, enfin, 9) dans une volonté globalisante, la dénonciation et la réénonciation critiques et esthétiques des grands paradigmes préexistants et, au premier chef, de la postmodernité elle-même, dans laquelle le sujet ne s'inscrit jamais aussi bien que lorsqu'il refuse ouvertement d'y prendre place.

Malgré cette fragmentation sèche de la postmodernité en neuf points - non fermés, il va sans dire - , il serait illusoire de penser lire ici des lieux de consensus cimentant l'unité d'un système. La postmodernité a sans doute ceci de singulier qu'elle n'investit jamais un lieu sans le transformer en un espace hybride de représentation, de réflexion, de dénonciation, de carnavalisation, de simulation ou de fictionnalisation. Ces espaces ne sont pas tant des modes de mise en discours que des figures qui informent le texte, inscrivant la position critique à l'intérieur même de la parole énoncée.

Ici, un arrêt sur image s'impose, car le titre de ce texte appelle des réflexions que cet article jusqu'ici ignore, déroulant un "paradigme critique " aux contours peu apparentés à ceux d'une québécité traditionnelle. Les visées performatives évidentes de toute spécification nationale - c'est-à-dire créer une identité opératoire - s'accordent en apparence fort mal avec un tel projet ludique, fictif, autoréflexif et tourné vers la mise en action à vide de ses propres rouages. Inscrite dès ses débuts dans le courant d'une modernité puis d'une postmodernité, la critique dite québé- 
$\operatorname{coise}^{6}$ semble donc soumise à des enjeux contradictoires où condition identitaire et condition paradigmatique s'affrontent, complexifiant dès lors les règles de fondation d'une pratique bercée entre ses impératifs nationaux et critiques.

D'entrée de jeu, une rectitude critique de bon aloi nous obligerait d'ailleurs à mettre en doute ici tout paradigme. Ou, plus précisément, toute autonomie paradigmatique de la critique québécoise car, si la littérature québécoise semble avoir su assez rapidement se distancier de la métropole française et faire de son écriture une pratique autonome, il ne semble plus aussi évident que la critique québécoise ait su faire de même, se définissant encore et toujours davantage par l'identité de son objet (la littérature québécoise) que par l'identité de sa propre parole ${ }^{7}$. Doit-on parler de la critique québécoise comme d'un sous-ensemble spécifié mais poreux d'un paradigme critique plus large, hésitant sans cesse entre sa dépendance et son indépendance, sa partition ou sa participation? Autour de quel lieu du discours doit-on

6 Soit celle des années 1960 et plus, c'est-à-dire l'époque pendant laquelle le mot "québécois " - autour duquel se cristallisent les arguments de l'identité ainsi que la consistance d'un nouveau "noyau" paradigmatique - prendra force de loi.

7 Le titre d'actes d'un séminaire récent (CRELIQ) sur la critique littéraire québécoise est lourd de sens à cet égard: dans Pour un bilan prospectif de la recherche en littérature québécoise (François Dumont et Louise Milot, Nuit Blanche éditeur, 1995), le mot nationalement marqué est bien "littérature = et non "recherche". Selon Lucie Robert appelée à réfléchir sur la question, "l'instauration d'une tradition nationale de recherche dans tous les domaines des sciences humaines et en particulier pour la littérature" (p. 35) demeurerait encore aujourd'hui à l'état de projet. Et, déplaçant l'épithète depuis l'objet vers la parole qui le réénonce, elle affirme qu'all est impératif d'assurer le développement d'une recherche québécoise sur la littérature [...]: le terme "québécois" est ici le mot important et renvoie au développement et à l'affirmation du caractère autonome de cette recherche" (Ibid.). Ici, l'exemple est prosaïque, mais montre bien comment le mot "québécois" pourra osciller entre deux lieux de définition, tantôt lié à celui d'un objet de discours, tantôt à celui d'un sujet. Paradoxalement, si l'on recule jusqu'aux premières années du discours dit québécois, il semble que le mot "québécois" ait d'abord été mis en discours dans des contextes argumentant l'existence d'une critique, d'une pensée, et non d'une littérature: le mot aurait désigné un sujet, un point de vue sur le réel avant que de surdéterminer l'objet du regard (voir Nicole Fortin, Une littérature inventée, Québec, Presses de l'Université Laval, p. 301-306). Il serait trop court de parler de volte-face ou d'abandon d'un projet initial d'abord inscrit autour de la figure critique; il faut plutôt dire que la surdétermination nationale pourra osciller d'un objet à l'autre, témoignant d'une complexité du système que j'esquisserai plus loin. 
14

formellement fonder les arguments d'un écart paradigmatique possible: dans les circonstances socio-historiques de son apparition (soit celles du renouvellement idéologique et discursif des années 1960-1970); dans l'identité (réelle ou construite) de l'énoncé littéraire ou de l'énonciation critique des discours; dans l'identité d'une médiation (théorique ou non) entre énonciation et énoncé; dans la facture formelle (figurative, stylistique, argumentative, générique, etc.) des discours critiques réalisés; dans le positionnement à l'égard de l'Autre (français, américain, canadien-français de jadis, etc.); dans la construction réflexive de sa propre image critique ou, encore, dans tous ces lieux à la fois?

Apposé au mot paradigme, le concept "critique" n'est pas plus univoque et l'on aura tôt fait de noter les ambiguités inhérentes d'une pratique critique québécoise où la constitution d'un "sujet" (d'un lieu métadiscursif de parole) s'est plus d'une fois heurtée à des rapports équivoques entretenus à l'égard d'un "objet. (littéraire, social, etc.) auquel il demeure historiquement, émotivement, discursivement et épistémiquement uni par des liens de proximité. Souvent plus près de l'inscription subjective que de la distanciation objectivante, souvent plus près d'un rapport à l'écriture propre à l'essai que d'un rapport au savoir propre à une critique théorisante, la critique québécoise semble, selon toute apparence, participer simultanément de deux paradigmes de discours concomitants, s'indexant à la fois comme écriture et comme lecture, comme discours et comme métadiscours - sinon aux seconds par l'exercice nécessaire des premiers.

S'il faut émettre ici quelque hypothèse permettant de cerner la possible singularité paradigmatique du modèle critique québécois - car je pose d'emblée, quitte à m'amender un jour, que la construction de ce modèle tend ici vers celle d'un paradigme spécifié - , force sera de dire que ce brouillage démultipliant les lieux d'inscription et d'institution ne relève pas des failles d'un système encore mal stabilisé. La critique québécoise trouverait plutôt les marques de sa distinction dans le positionnement ambigu de son sujet ou, plus précisément, en se faisant le discours de l'ambiguité identitaire, fondant son écriture - en ses niveaux énonciatif, figuratif, argumentatif, etc. - sur l'inscription d'une bétéroglossie et d'une bétérodoxie critique qui démultiplient les voix, les codes, les sources, les genres. Notamment, contrairement à d'autres modèles, la critique québécoise ne se constituera 
jamais autour d'une forme théorique objective: nulle théorie spécifiquement québécoise, avoueront d'aucun, n'a jamais clairement émané de cette pratique, avant tout construite par divers discours théoriques d'emprunt qu'elle recatégorise selon les stratégies de son propre discours. La critique québécoise semble en effet se déployer comme un espace de recyclage et de réénonciation, qui construit sa distinction critique en phagocytant d'autres discours. Surtout ne faut-il pas conclure à quelque manque d'" imagination critique ", mais plutôt croire que c'est en réénonçant différemment (et souvent subjectivement) l'Autre que la critique québécoise pose d'abord son écart, sa distance et son identité face à ce qui est extérieur à elle-même.

On rappelera ici l'origine du mot paradigme ( $\pi \alpha \rho \alpha \delta \varepsilon \gamma \mu \alpha)$ où $\delta \varepsilon \gamma \mu \alpha$ signifie "montrer" et $\pi \alpha \rho \alpha$ "à côté" ou "en comparaison": les emprunts au(x) paradigme(s) de l'Autre ne veulent donc pas seulement dire participation (ou aliénation, pour reprendre un jugement cher aux années 1960); ils participent également d'un positionnement permettant d'établir, figurativement et argumentativement, sa propre logique et sa propre distinction paradigmatique. En fait, et ainsi que j'entends l'illustrer dans la suite de ce texte, il serait plus juste de dire que cette critique se constituera sur une cobésion énonciative, fondant sa distinction en donnant forme à un "sujet critique" spécifié. Le paradigme critique québécois ne se serait pas institué par la constitution d'une position d'objectivité théorique autant que par celle d'une subjectivité prise au sens d'inscription du sujet, et non d'émotivité - dont on peut retracer les modes et les étapes de mise en place à l'intérieur même de l'énoncé critique, et ce, depuis les années 1960 jusqu'à aujourd'hui.

À cet égard, la détermination québécoise du discours ne délimiterait pas seulement un espace géographique et historique de parole: le mot ne rompt pas seulement géographiquement avec "français " ou historiquement avec "canadien-français", servant à localiser le ici-maintenant d'un nouveau paradigme; il détermine et nomme davantage un sujet spécifié, ou une position discursive inédite, concourant d'un même souffle à surdéterminer la critique elle-même. Donnée de base du travail identitaire et unitaire par laquelle se fonde la différence du discours, la québécité n'en est pas moins une notion mobile qui peut être variablement investie selon les lieux de sa mise en discours: plus d'une forme d'indexation paradigmatique pourrait en effet légitimement la 
revendiquer. La québécité, vue comme identité intellectualisée par le discours ${ }^{8}$, peut fort bien se concevoir comme une notion romantique en ce qu'elle est un lieu de positionnement et de définition d'un sujet autonome - d'un "je" ou d'un "nous" -, concrètement manifesté par les notions d'identification à l'œuvre, d'appropriation réelle et émotive du monde, de rupture nostalgique face au passé, d'inscription dans un ici-maintenant du texte et du contexte. Mais cette québécité intellectualisée peut aussi se présenter comme une notion profondément moderne en ce qu'elle vise la construction d'un objet-nation autonome duquel il faut objectivement et "objectalement" se distancer et l'envisager scientifiquement et structurellement - comme société, comme texte, comme corpus, comme institution, comme culture. Cette québécité, enfin, peut bien être lue comme une notion postmoderne en ce qu'elle est intimement liée à sa propre négation et ironisation, se définissant moins comme objet ou comme sujet que sous le mode d'une impossibilité foncière à objectiver ou subjectiver. Fondée sur l'altérité, sur l'extériorité, sur l'ambiguité et l'hétéroglossie de ses codes, cette québécité se maintient comme discours, ou comme lieu de discours, en tant qu'espace sans cesse dénoncé, transgressé, aboli ${ }^{9}$. Dès lors, cette cohérence québécoise existe moins comme identité - comme lieu identitaire du sujet et de l'objet - que sous la forme d'une pratique d'identification, d'un lieu de questionnement d'une identification se suffisant à luimême, hors de toute recherche transitive et nécessaire de résultat.

Cette vision en triptyque, à coup sûr métaphorique, est d'autant plus complexe que, bien que ces trois étapes de la critique puissent être historiquement saisies en succession - se parta-

8 Est-il en effet utile de préciser que pareille notion de québécité peut - sinon doit - être considérée comme une construction de discours, liée à des schèmes d'intelligibilité, à des modes de représentation référentiels ou imaginaires, à des consensus d'intellection qui ordonnent la forme du réel.

9 Je ne nommerai qu'un des textes célébrés de ce mode d'inscription du signe national, à savoir L'écologie du réel. Mort et naissance de la littérature québécoise contemporaine (Pierre Nepveu, Boréal, 1988): la réindexation du sens énoncée dès le titre - voir l'inversion de "mort" et de "naissance" - se trouve confortée dès la première de couverture, où l'on annonce qu'il s'agit bien de « Repenser le mode d'être de la littérature et de la culture québécoises, moins en tant que littérature et culture nationales qu'en tant que contemporaines, sur un fond de catastrophe et d'étrangeté, d'éclatement et de burlesque. " Pour l'analyse de ce texte, j'invite bien sûr le lecteur à lire un des textes du présent numéro, où les stratégies de cet ouvrage sont analysées en détail. 
geant les décennies 1960, 1970, puis 1980 et suivantes - il serait plus exact de dire que ces trois modes de conceptualisation des signes nationaux se sont toujours concurremment inscrits dans le discours critique. De plus, en reprenant ainsi ces trois grands paradigmes préconstruits du savoir - lisibles comme paradigmes réels mais aussi comme paradigmes rêvés sur lesquels plus d'un discours savant contemporain se fonde dans sa traversée du parcours, en partie imaginaire, des modèles critiques ${ }^{10}$-, mon intention est principalement de montrer que la critique québécoise s'est constituée, sur trente ans, sur une logique conventionnelle, sinon mytbique, de constitution du sujet critique. En effet, ces grandes étapes ou époques du savoir procèdent peut-être avant tout d'une représentation du réel critique à laquelle aucune critique en instance de définition ne peut cognitivement échapper: dans toute perception de la critique en tant que paradigme, ces mots désignent plus que des dates; ils prescrivent des trajets, des trajectoires, des virages, des sens interdits, etc. Entre autres, dans un geste de réindexation typique à la postmodernité, Jacques Allard pouvait déclarer que "l'année 1960 constitue pour beaucoup le point pivotal; avant, c'est moderne (depuis 1895 ou 1900 ) ; après c'est postmoderne "11. Pareils propos sur la constitution littéraire pourraient servir à désigner la constitution critique québécoise qui, dès ses premiers jours, a eu, d'une part, à poser les pivots de son évolution et, d'autre part, à se "positionner" comme espace d'énonciation par la dénonciation et la

10 Une étude du sens général donné par les discours à l'évolution épistémique du savoir aurait sans doute tôt fait de démontrer que ces trois paradigmes construits ne sont pas seulement des étapes empiriques, mais bien des représentations a posteriori, qui nous en apprennent long sur l'imaginaire d'une critique intéressée par la visualisation de son propre parcours. Ce rabattement et cet usage de ces grandes étapes usuelles de la constitution - ou de la destitution - du sujet (critique) sont notamment opérés dans un texte que j'ai déjà cité dans cet article, soit celui de Walter Moser (op. cit.) où sont reconstruites la logique et l'évolution critique autour des trois formes de la modernité, du romantisme et de la déconstruction. On pourra notamment y noter l'" inversion " des deux premières étapes par rapport à la représentation habituellement donnée de l'histoire épistémique du savoir, inscrivant pareilles étapes dans un travail de reconstruction du parcours critique: comme l'avoue le critique conscient de sa réécriture, cet alignement "n'a ici qu'une valeur heuristique et ne correspond pas nécessairement à un déroulement historique réel. Bien au contraire" (p. 127).

11 Jacques Allard, Traverses de la critique littéraire au Quêbec, Montréal, Boréal, coll. "Papiers collés", 1991, p. 94. 
réénonciation de modèles critiques antérieurs ou étrangers. C'est entre autres à partir d'un tel travail que la critique nationale a su inscrire les marqueurs de son identité et de sa différence.

Selon cette logique, il ne s'agit donc pas de soumettre ces étapes épistémiques aux vogues théoriques qui purent historiquement avoir cours : la forme de l'énonciation (l'ancrage formel du "je" dans le texte savant) et l'argumentation de l'énonciation (l'ancrage réel du "je" dans le contexte savant) sont deux lieux de mise en discours parfois inscrits dans des parcours aux tracés autonomes ${ }^{12}$. Mon tracé des paradigmes critiques se situera davantage du côté de l'inscription formelle du parcours énonciatif que du côté de son argumentation empirique, liée aux modes et aux contextes de parole dominants.

Eu égard à ces données, les cas les plus intéressants seront sans doute ceux où l'adoption d'un appareillage méthodologique ne renforce pas seulement l'argumentation du texte (recherche de scientificité, d'indexation à un lieu et à une tradition de savoir sanctionnés) mais agit comme signifiant, c'est-à-dire comme structure régulatrice qui prescrit l'inscription formelle du sujet de l'énonciation. Au Québec, on pourrait notamment citer le cas de l'inscription de Bakhtine dans l'écriture d'André Belleau: chez ce dernier, l'usage des notions de dialogisme, de conflit des codes, de carnavalisation, etc. ne servent pas seulement à situer ni à fonder un mode savant d'interprétation de l'objet littéraire; plus intéressante, parce que moins explicite, est sans doute l'inscription de ces concepts dans la structure énonciative adoptée par le critique. Dans Surprendre les voix - recueil posthume où le choix et l'ordre des textes tracent en quelque sorte un paradigme ou, du moins, un nouvel axe de lisibilité dans le parcours critique d'un "je" 13 -,

12 Par exemple, un énoncé critique, dont l'argumentation relève de l'appareillage théorique de la modernité, peut fort bien donner lieu à une formalisation "romantique" du sujet de son discours. Il en est ainsi des premiers discours québécois qui seront parcourus par le discours telquélien: bien que théoriquement plus "modernes" et "branchés "que leurs prédécesseurs - la nouveauté étant ici un effet recherché et explicitement inscrit dans les discours -, nombre de ces discours demeureront traversés par les mêmes stratégies d'inscription du sujet de l'énonciation que celles préconisées par les discours anciens. Les étapes du discours ne sont donc qu'accessoirement liées aux "évolutions" théoriques en cours.

13 André Belleau, Supprendre les voix, Montréal, Boréal, coll. "Papiers collés", 1986. Pensé par Belleau, ce recueil ne paraîtra qu'après le décès de l'auteur 
l'exemple le plus fort de cette indexation de Bakhtine dans la forme même du texte apparaît sans doute dans les premier et dernier textes, respectivement intitulés "Mon coeur est une ville" et "Lorsqu'il marrive de surprendre des voix". Ressemblant davantage à des fictions autobiographiques qu'à de purs essais ${ }^{14}$, ces deux textes fragmentent l'énonciation, présentant des je multiples posés tantôt en parallèle, tantôt en conflit, mais toujours dans une hétéroglossie, dans une polyphonie qui représente - au sens mimétique comme au sens carnavalesque du terme - le champ des tensions que déploie la représentation bakhtinienne. Dans le premier cas, deux paroles et deux parcours du savoir s'esquissent, inscrivant ici le trajet d'un $j e$ en quête de Montréal, traçant là (dans un texte formellement démarqué par l'italique) la voie d'un autre je-sinon le même - à la recherche d'une femme. Dans le second cas, le texte se construit plutôt comme le dialogue de trois voix en désaccord, voix qui, à la fois, parlent de, parlent à et sont Belleau: "mon "je", dit l'une d'elles, est multiple et hétérogène. Il [Belleau] n'arrivera jamais à lui seul à me sommer et à m'unifier. "15 Véritable mise en abyme exacerbée des débats du sujet et des codes que la théorie donne à lire dans l'objet littéraire, cette formalisation singulière de l'énonciation soumet donc la théorie - ou toute argumentation d'autorité - à la formalisation d'une parole critique, et non seulement à l'objectivation de l'objet littéraire analysé.

Par voie de conséquence, que le premier de ces textes ("Mon cœur est une ville", 1959) précède chronologiquement toute introduction formelle ou argumentative de Bakhtine dans

et sera reçu, par plus d'un lecteur critique, comme un testament critique donnant un reflet modèle et complet de l'itinéraire intellectuel de l'essayiste. Il faudra un jour étudier la réception de Belleau par ses analystes: non seulement parce que son œuvre y fut jugée comme apport majeur dans la tradition critique québécoise; surtout parce qu'il y sera toujours l'un des premiers critiques québécois (d'après 1960) à avoir pu être lu sous la forme d'œutre complète, donc fermée et lisible comme représentation exemplaire d'un parcours critique global, depuis ses débuts jusqu'à ses dernières réalisations. Je remercie Annie Perron qui, dans le cadre d'une récente rencontre du CRELIQ (Laval), m'a conduite à me repencher sur ce texte de Belleau.

14 Les autres textes du recueil entreraient davantage dans la catégorie générique de l'essai. Ce qui est dit ici à propos des textes d'introduction et de conclusion - qui, par leur position, ne sont pas sans raison ceux qui «ictionnalisent . le plus les stratégies énonciatives - aurait néanmoins pu s'appliquer à autres car l'énonciation s'y inscrit selon des visées similaires.

Ibid., p. 227. 
l'écriture du critique ne peut pas être perçu comme un anachronisme. On pourrait plutôt y trouver la confirmation que le recours à la théorie n'advient pas seulement comme un argument, comme une grille extérieure importée par une instance énonciative pour donner forme à l'objet; elle peut survenir comme réponse, comme forme, comme sanction d'une structure déjà prédéterminée par le projet d'écriture critique. Dès lors, le texte critique semble se présenter comme projet de constitution d'une énonciation, énonciation dont on doit assurer l'inscription, mais aussi l'objectivation et la signification - à l'image des étapes successives du parcours par lesquelles le romantisme, la modernité et la postmodernité ont peu à peu su saisir et formet le sujet.

De la sorte, que l'essai, plutôt que le texte théorique pur, ait été la forme la plus usuelle de l'écriture critique québécoise n'est sans doute pas qu'une question de refus de quelque posture calquée sur le modèle scientifique. Lieu de constitution du sujet, l'essai se profile sans doute comme forme de prédilection, parce qu'il pose non pas le parcours critique dans l'inscription argumentative d'un position d'objectivité mais plutôt dans l'inscription formelle d'une subjectivité, d'une énonciation. Plus précisément, la critique québécoise se pose, depuis ses premières paroles, non pas comme un discours critique institué (socialement), mais d'abord comme une pratique qui a à mettre en discours, à gérer et à instituer de l'intérieur les règles générales de sa condition critique, voire de sa condition paradigmatique.

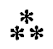

Formellement, trois types de mise en discours de la position critique québécoise pourraient donc être avancés: 1) inscription; 2) objectivation et 3) signification. Ces trois étapes, énoncées ici pour leur valeur heuristique plus que pour leur portée historisante, viseront au repère des divers dispositifs de formation d'une énonciation critique québécoise qui acquiert, à travers leurs actions, une forme de plus en plus constituée. Pour qu'elles puissent espérer être opératoires ${ }^{16}$, ces étapes devront être vues

16 La typologie présentée, qui trouve ses assises dans Une littérature inventée (op. cit.) où je m'attardais essentiellement à l'étude des années 1965-1975, se veut une étape préliminaire d'une recherche plus large où il s'agira de saisir 
comme trois moments aux frontières malléables, mais qui sauront rendre compte de la dynamique constitutive d'un paradigme critique québécoise, à travers lequel la critique a construit successivement sa consistance institutionnnelle: 1) d'abord par l'inscription discursive nécessaire, dans l'énoncé critique, d'un sujet de l'énonciation, ensuite 2) par l'objectivation de cette position, garantissant la reproductibilité de celle-ci dans d'autres séries de circonstances discursives, et enfin 3) par la signification octroyée à cette position, permettant de donner valeur, usage mais aussi sens rétroactif à un parcours critique pris dans son ensemble.

\section{1) inscription}

Tout discours critique, même le plus enclin à couvrir sa parole du masque de l'anonymat savant, ne peut s'abstraire d'un ancrage énonciatif nécessaire. On le sait depuis longtemps, l'objectivité ne saurait signifier quelque absence du sujet dans le discours. Celle-ci rend plutôt compte d'une forme énonciative particulière où le je - converti par le point de vue théorique adopté, par l'englobement de la parole dans la collectivité d'un nous (mis pour chercheurs, compatriotes, etc.) ou par diverses stratégies d'effacement et de travestissement - se dévoile dans ses rapports avec le champ de discours dont il est, à la fois, le tributaire et le gérant. En critique comme en science, ce que l'on appelle l'objectivité ne correspond pas tant à la soumission de l'énonciation aux stratégies d'un énoncé qu'aux premiers signes qu'il existe, hors du $j e$, un lieu idéal d'où peut adéquatement s'énoncer le savoir. Bien qu'elle crée l'illusion d'un retrait du je, cette objectivité ne peut qu'impliquer l'existence d'un paradigme, soit d'un modèle suffisamment cohérent pour autoriser tout éludation du sujet singulier. En ce sens, autant la subjectivité que l'objectivité des discours peuvent se lire comme lieu d'une mise en place des assises nécessaires à l'émergence d'un paradigme critique donné. C'est autant dans la proclamation que dans l'effacement du sujet que peut se lire l'embryon d'une pratique spécifiée et autonome.

Ainsi, parler de stratégies d'inscription de l'énonciation ne vise pas tant à signaler la présence d'un je totalement incapable,

les grandes étapes discursives de constitution d'un paradigme critique québécois. Mes propositions d'aujourd'hui seront donc volontairement exploratives et sujettes à de futures nuances. 
de toute manière, d'être absent: plus intéressant serait de saisir les modalités singulières de cette inscription. Que le je soit présent par des énoncés tels que "à mon avis", "mon analyse", "j'énonce que" ou qu'il soit travesti sous des formules telles que "plus d'un chercheur pense", "selon la théorie", "le texte se donne à lire", l'on peut toujours présumer qu'une énonciation singulière agit dans chaque texte: énonciation autour de laquelle se construit peu à peu une position de lecture normalisée régularisant la pratique critique dans son ensemble.

Un regard sur la critique littéraire québécoise des débuts permet en effet de noter que la constitution du "je" s'est faite en partie par l'ancrage formel (plutôt qu'institutionnel) de ce dernier: l'adoption quasi généralisée de la forme générique de l'essai ou de la critique, définie comme pratique en opposition à toute forme objectivante liée à l'usage des théories - doit d'ailleurs être envisagée comme l'un des indices formels de cette action, permettant d'asseoir dans la forme, plutôt que dans l'usage, les normes d'une pratique. On peut d'emblée avancer que la critique québécoise est sans doute plus générique - soit liée à l'inscription d'une norme de discours et d'écriture - que soumise à la normalisation d'un grille de lecture objective et préconstruite: "La critique, disait-on déjà en 1966, situe l'œuvre à tel ou tel niveau d'existence littéraire moins par les jugements de valeur qu'elle formule que par la façon dont elle en parle. " ${ }^{17}$ Et c'est en la concevant comme norme d'écriture - ou comme distinction discursive - qu'il faut en effet saisir les modes d'inscription de l'énonciation québécoise et, partant, de la position critique.

Dès lors, s'il s'agit de débusquer les normes singulières d'une inscription énonciative, force sera de reconnaître que, dès le début des années 1960, certaines formes de mises en discours parcourront plus d'un texte, esquissant un modèle de discours stéréotypé et reproduisible. L'entrée dans l'ordre du discours n'y est pas inconséquente, comme en témoigne nombre d'introductions aux profils analogues: "il est difficile de parler d'un poète d'une façon pertinente" 18 ; "La première phrase de L'biver de force, véritable sommaire de tout le récit, n'est pas plus intelli-

17 Laurent Mailhot, "Une critique qui se fait", Études françaises, vol. II, no 3, 1966 , p. 346 (l'italique est de moi).

18 Noël Audet, "Structures poétiques dans l'oeuvre de Fernand Ouellette", Voix et images du pays III, 1970, p. 103. 
gible à la centième lecture qu'à la première " ${ }^{19}$; "nous sommes habitués à lire des œuvres plus rassurantes -, notre première réaction consiste, soit à la rejeter, soit à lui donner une orientation trop précise (ce qui constitue une autre façon de trahir la parole)." ${ }^{20}$ Ici, que le je soit figuré ou non, l'énonciation s'inscrit néanmoins autour d'invariants: il ne s'agit pas de poser la compétence du sujet ni quelque posture d'autorité, mais bien de définir d'emblée les limites ou les difficultés d'une lecture autre, que la suite de l'article contribuera invariablement à remplacer par une démarche plus efficace. Pareil modèle parcourra aussi les conclusions des textes, dans lesquelles est sanctionné le dépassement des limites de lecture initiales ${ }^{21}$ : la sanction du discours critique s'axe donc moins sur l'objet littéraire que sur le sujet critique lui-même. Plus précisément, la position critique ne se construit pas en validant d'emblée son efficience énonciative mais en construisant, par diverses stratégies de mise en discours, l'espace et les règles de son propre exercice.

D'où les observations suivantes. a) Ce n'est pas sans but que j'ai usé du mot sanction, cher au parcours narratif greimassien. Il ne faut pas juste voir la sanction comme un jugement de valeur (argumentatif et axiologique) mais bien comme l'aboutissement d'un parcours (narratif) invariablement fondé par des étapes construites à même le discours. En découle une première observation, à savoir que la position énonciative, bien qu'objet d'une inscription argumentative, est plus foncièrement l'objet d'une inscription narrative: le texte québécois des débuts ne cherche pas tant ses arguments hors du discours ${ }^{22}$ que dans la représentation,

19 Georges-André Vachon, "Note sur Réjean Ducharme et Paul-Marie Lapointe (Fragment d'un Traité du vide)", Études françaises, vol. XI, $\mathrm{n}^{\mathrm{Ds}} 3-4,1975$, p. 356.

20 Yvan Lajoie, "Roland Giguère, à la recherche de l'essentiel ", Études littéraires, vol. V, $\mathrm{n}^{\circ} 3$, décembre 1972, p. 411.

21 La conclusion suivante illustre un tel parcours évolutif, comme en témoigne les mots "chronologiquement", "passer de ", "conduisant ", "partis de ", "devenons", "progressiven: "Chronologiquement notre lecture [...] nous fait passer d'une antitude de passivité, d'un sentiment d'impuissance à un dynamisme conduisant à la violence. Partis d'une position de retrait, d'absence par rapport à la réalité du monde des hommes, nous devenons témoins d'une acceptation progressive et d'une prise en charge de cette réalité "(Richard Giguère, "D'un "équilibre impondérable" à une "violence élémentaire" ", Voix et images du pays VII, 1973, p. 90).

22 Soit, par exemple, par des citations d'autorité ou par des références à des théories reconnues qui sont autant d'énoncés externes qui, par l'espace 
à l'intérieur de l'énoncé, des étapes ${ }^{23}$ de constitution de la position du sujet critique. b) Transformer ainsi l'espace du texte en un espace de représentation et de fictionnalisation n'est pas sans conséquences. Au terme du procédé, la lecture - ou l'analyse, ou la critique, selon la forme que l'on choisit - ne devient pas qu'une pratique critique qui trouverait, dans l'écriture, un de ses lieux d'exercice; elle est bien une figure créée par et dans le discours. Les théories, les discours d'autorité, les consensus, les polémiques, etc. n'y résultent pas d'abord d'une institution présuposée et située bors discours mais trouvent plus profondément leur usage et leur valeur par la spécificité de leur mise en discours.

Dès lors, ce discours québécois n'est pas tant le lieu d'inscription d'une énonciation singulière qu'un espace de représentation, de fictionalisation, et partant de constitution d'énonciations multiples et hiérarchisées: elle mettent donc en scène les forces qui cimentent tout paradigme critique. Ici, l'énonciateur, même le plus assuré de sa compétence, n'avance jamais seul dans le texte et amène avec lui toute une série d'énonciations possibles ou impossibles, valorisées ou disqualifiées, concourant dès lors à

référentiel (d'origine contextuelle ou co-textuelle) d'où ils proviennent, permettent d'inclure celui qui les cite dans l'espace référentiel généralisé - soit, par extension, le paradigme - de la critique.

23 Cette représentation peut être mise en abyme et être investie par des apersonnages". C'est le cas dans ce paragraphe d'introduction où se voit narrativisée l'action du mauvais lecteur, d'ailleurs remplacé, dans la suite du texte, par une figure de lecteur plus conforme aux enjeux critiques nationaux: "Celui qui s'obstine à lire Les anciens Canadiens comme un roman risque cependant, à première impression, d'être déçu. Il se heurte à une prose touffue et naïvement pédante, il s'égare dans les méandres du récit fantaisiste des événements de 1760 , bref il cherche péniblement une cohérence à ce chatoyant galimatias. Perplexe, il interroge les primitives statues que sont les personnages et se met à scruter le texte à la recherche de significations symboliques" (Nicole Deschamps, "Les "Anciens Canadiens" de 1860. Une société de seigneurs et de va-nu-pieds", Études françaises, vol. I, n 3, 1965 , p. 3). Cette représentation peut aussi parcourir le texte à travers des énoncés qui narrativisent les différentes étapes de la lecture: "Nous azons essayé de montrer un enchaînement entres les images et les thèmes principaux de "Vivre mieux", (selon un schéma linéaire de lecture)"; "nous avons constaté qu'ils peuvent se regrouper [...]"; "Aussi avons-nous voulu éviter toute référence qui ne se rapportait pas directement au poème [...]"; "Nous avons pu dégager une ligne de force essentielle qui nous permettra de mieux situer le poème" (Yvan Lajoie, op. cit., p. 419; l'italique est de moi). 
poser les premières règles d'un système complexe d'interrelations entre différents points de vue sur la littérature. La pluralité des locuteurs, la co-présence d'énonciations modernes et anciennes, l'importance accordée à l'inscription d'une "tradition de lecture" (Georges-André Vachon), le positionnement à l'égard de l'altérité (française), le refus d'une démarche purement "objective", etc. témoignent, en effet, que la critique se définit ici et dès le départ comme une pratique généralisée et collective d'énonciation.

S'il faut parler d'une première étape pendant laquelle la critique québécoise a cherché à inscrire les modalités de son énonciation savante, force sera de dire qu'elle ne pose pas tant un sujet qu'un espace intersubjectif de parole, condition première à la mise en place d'un possible paradigme.

\section{2) objectivation}

La forme d'objectivation la plus usuellement présente dans les paradigmes du savoir réside sans doute dans la théorie: non parce qu'elle est garante d'objectivité ; plutôt parce qu'elle objective, c'est-à-dire transforme en objet un point de vue qui devient, en quelque sorte, le lieu d'énonciation du texte. Lieu d'énonciation dont l'avantage notable est d'être reproduisible, réitérable dans diverses circonstances: dans sa participation à un paradigme, la théorie a l'atout majeur d'assurer une certaine homogénéisation du point de vue et, par conséquent, une certaine unité identitaire contrant l'altérité de je multiples. Les faits nous apprennent qu'il ne s'agit sans doute pas là du mode privilégié par la critique québécoise et l'on pourra trouver, dans les conditions même de l'inscription de l'énonciation québécoise, les raisons de cette singularité. D'abord tournée vers l'inscription et la représentation d'un lieu de polyphonie énonciative, la critique québécoise semble en effet avoir conforté sa pratique en transformant en objet le champ hétérogène de ses discours sur la littérature: l'importance accordée aux théories de l'énonciation, de l'institution $^{24}$, du discours social, de la réception, etc. témoigne d'une

24 L'institution est "la mise en abyme de la deixis; [elle] est le récit onomastique de la deixis, du je-ici-maintenant de l'institution (littéraire) [...] le récit de la deixis n'a cesse de se modeler, de se moduler et de se modaliser selon toutes sortes de tours, de détours et de retours, institutionnels ici énumérés: édition, promotion [...], enseignement, recherche... " Jean-Marc Lemelin, 
volonté d'objectivation de différentes formes d'énonciations, qu'elles soient individuelles, sociales, idéologiques ou autres 25 . L'aspect théorique ou objectif de ces méthodes a sans doute moins d'incidence que le fait que, dans tous les cas, celles-ci cherche moins à définir les formes de l'objet littéraire que celles des instances qui les énoncent, qui les régissent et qui deviennent dès lors les véritables objets sur lesquels se penche l'analyse.

Les stratégies d'inscription décrites plus haut ne feront en effet qu'un temps. Elles se verront bientôt remplacées par des discours où l'énonciation n'est plus vraiment l'objet d'une mise en parcours d'où elle sort sanctionnée et située par rapport au champ des autres énonciations possibles. S'il faut parler d'une seconde étape pendant laquelle la critique québécoise a cherché à consolider et à objectiver les modalités de son énonciation savante, force sera de dire qu'elle réside dans une certaine mise à l'écart, où le lieu de la parole critique n'est plus tant un sujet qu'un objet de discours. Préservant un certain éclatement dans les formes que pourra prendre l'énonciation - éclatement que confirme l'adoption toujours effective de discours de type "essai" - , la critique québécoise semble plutôt avoir construit sa consistance paradig-

"Les paradoxes de l'institution (littéraire). Études et attitudes littéraires", dans Robert Giroux et Jean-Marc Lemelin (dir.), Le spectacle de la littérature. Les aléas et les avatars de l'institution, Montréal, Triptyque, 1984, p. 207). L'institution, dont les applications théoriques à la littérature ont sans doute été plus populaires au Québec qu'en tout autre lieu, n'est peut-être pas tant une façon matérialiste de concevoir la littérature qu'une façon de systématiser, d'objectiver les différents de l'énonciation du littéraire. Selon un autre point de vue mais liant tout autant la réalité et l'itérativitē de l'institution à l'existence d'un lieu de discours, Gille Marcotte pouvait dire que "du moment que l'institution littéraire est dite, elle devient réelle et pèse d'un poids spécifique sur les activités qui ont lieu (qui auront lieu) dans son aire" ("Institution et courants d'air", Littérature et circonstances, p. 17).

25 J'ajouterai ici le cas des études féministes, dont les assises reposent intrinsèquement sur l'existence d'une altérité ou d'une spécificité énonciative dont on cherche à circonscrire le terrain d'exercice. Qu'une pareille démarche ait occupé et occupe toujours un pan important de la recherche québécoise ne peut pas être tenu pour accessoire, même lorsque l'on veut spécifiquement lire l'institution nationale de la critique. La présence simultanée de diffêrentes pratiques critiques tournées vers les questions identitaires donne peutêtre prise à des configurations, à des noyaux, à des topoï dont il faudra un jour cerner la dynamique. Tous les discours québécois sur l'altérité, qu'ils concourent ou non à définir le centre ou les marges d'une parole nationale, gravitent sans doute autour des mêmes lieux de questionnement et de solution. 
matique en transformant sa parole hétérogène en un objet de discours: on y décrit dès lors des postures, des lieux, des regards exemplaires et réitérables dans les autres circonstances de discours soumises au paradigme général de la critique québécoise.

Derrière mes propos, il ne s'agit bien sûr pas de comprendre que, au Québec, toute objectivation critique par la théorie - soit par la désignation d'un regard détaché de lobjet - sera totalement occultée et insignifiante: il faut peut-être plutôt concevoir que la posture théorique sera absente en tant qu'argument unitaire de la critique québécoise, la théorie ne prenant jamais la forme d'une "énonciation collective" pouvant indexer et conjoindre toutes les énonciations singulières tenues à un même instant dans un champ critique donné. La théorie ne serait pas, pour cette critique, l'argument paradigmatique fondamental, cette demière privilégiant plutôt des arguments d'ordre énonciatif et discursif.

\section{3) signification}

Devant pareille critique québécoise qui semble dévier de la trajectoire familière que l'on prête aisément aux paradigmes savants, donc devant pareille critique qui semble s'objectiver en posant comme lieu de discours sa propre parole et son propre texte, on ne peut sans doute que s'attendre à ce qu'une nouvelle étape s'inscrive, appelant la critique à se former en donnant sens - c'est-à-dire orientation et signification - à l'ensemble de sa trajectoire, de ses enjeux, de ses options ou de ses apories. Nombre des discours de déviation, de contestation, d'ironisation, de dépréciation, de fictionnalisation, etc. que ce procès critique adoptera pour parler d'elle-même pourraient sans doute entrer dans cette catégorie du miroir, où l'enjeu est de mettre en discours les conditions et les contradictions mêmes d'une discursivité critique. Advenant ici tel un bappy end - la fin de mon article ressemblant opportunément à son ouverture - cette étape (ultime) axée sur la signification des "modèles" n'est peut-être pas seulement conjoncturelle mais soumise au développement même de quelque paradigme. Son déploiement tendrait peut-être surtout à confirmer que l'identité, qu'elle soit liée à l'existence critique ou nationale des discours ou liée à celle de toute autre forme paradigmatique, peut prendre moins la forme d'un but visé que celle d'un lieu de discours, où les signes et les sens sont tour à tour mis en jeu et mis en scène. 